\title{
Numerical Simulation of Stress-Strain State of Thick Coal Seam at the End of Long-Pillar Work
}

\author{
Vitaliy Trofimov ${ }^{1}$, Sergey Kubrin ${ }^{1, *}$, Uriy Filippov ${ }^{1}$, and Igor Haritonov ${ }^{2}$ \\ ${ }^{1}$ EDP Institute of Comprehensive Exploitation of Mineral Resources Russian Academy of Sciences s, \\ Editorial Department, 111020 Krukovskiy tupik 4, Moscow, Russia \\ ${ }^{2}$ JSC SUEK-KUZBASS, 652500 Vasilieva 1, Leninsk-Kuzneckiy, Kuzbass, Russia
}

\begin{abstract}
The article deals with the redistribution of stresses and the formation of fracture regions at the final stage of the excavation post. Considered various options in the formulation of the problem, describe the solution in full and the additional stresses. Presented mechanical model describing the redistribution of the original stresses caused developing mining operations; formation of discharge zones and the additional load near the bottom of the lava and around the DC; areas of possible fall-outs of rocks; displacement of rocks, in particular faith-vertical in roof of recreation center and horizontal to vertical outcrops of in-fighting and dismantling the camera. The deformation model used in solving the problems is built taking into account the possible plastic deformation of both coal and the immediate roof of the formation, which allows taking into account both the fracture of the massif and the nonlinear deformation of the material itself. It is emphasized that the parameters of plasticity can be determined from the solution of the inverse problem using experimental data obtained in situ.
\end{abstract}

\section{Formulation of the problem of the stress state of the massif in the development of mining}

The practice of mining coal shows that in most of the excavation site the most dangerous areas are the places where the excavation site crosses previously passed workings, in particular dismantling chambers (DC) (the regional area completing the face processing) [17]. There are two main ways to prepare the treatment face for dismantling the equipment at the end of the work in the excavation site: the formation of the DC in the treatment face as the complex moves and the early formation of the DC with the help of tunneling equipment at the site of the future stop of the treatment works in the face. In the first case, the process of redistribution of stresses in the coal seam and rocks is continuous as the length of the layer is increased. At the same time, the vertical load on the boundary part of the formation ages, exceeding several times the initial vertical stress in the array. Further, the main roof and the underlying rocks gradually fall on the collapsed rocks of the immediate and false roofs, and thereby reducing the load on the marginal part of the formation. The initial

\footnotetext{
* Corresponding author: $\underline{\text { s_kubrin@mail.ru }}$
} 
increase in mountain pressure with the subsequent decline is due to the change of the regime of interaction between the roof and the soil of the treatment space [6-7]. In the second case, when DC is created in advance, there is all the above, but in this case there is an additional stress concentrator in the marginal part of the formation in the form of a chamber with partially anchored walls and roof. At the approach of excavation site to the dismantling chamber there are difficulties associated with the possible collapse of the roof and extraction of coal from the face. Gradually decreasing the pillar between the DK and the control space can be fragile and unmanaged collapse.

Let us consider the General formulation of the problem of assessing the change in the stress-strain state of the massif associated with an increase in the developed space in the process of mining a horizontal coal seam. The parameters of the testing system make it possible to adopt a flat design model. At the same time, it was considered that the depth of the development of the horizontal layer is $H=130 \mathrm{~m}$ thickness of a seam $m=4.5 \mathrm{~m}$. in the presence of DK, its size is $4.5 \times 4.5 \mathrm{~m}$, the size of the pillar $L$ varied within $36 \mathrm{~m}-0 \mathrm{~m}$. As the excavation site progresses and approaching it to DC, i.e. with a decrease in the pillar, its loading gradually increases to a value determined by the limit of plasticity of coal and at $L \sim 30 \div 40 \mathrm{~m}$ reaches a maximum value. Its value is over-hanging from the properties of the rock massif and coal seam and typically $2.5 \div 3 \gamma H$. For more soft and plastic coal it is smaller, and for more elastic - more. When further promoting the slaughter of destruction cover all large part of the rear sight and it gradually pillar its carrying capacity and its loading decreases, which has been shown in [7].

With the development of mining, the vast expanse is filled with rocks of collapsed false and immediate roof, and possibly overlying rocks. In the calculations, it was modeled by an elastic body with a modulus of elasticity an order of magnitude smaller than that of coal.

More precisely, the value of this module $E_{c o l}$ can be determined from the solution of the inverse problem. For this purpose it is necessary to focus on two extreme cases: the first there are no collapsed rocks and the second - the properties of collapsed rocks coincide with the properties of the genus of the roof array. In the first case, the lowering of the earth's surface at full landing will be equal to the thickness of a seam $h$, while $E_{c o l}=0$ can be assumed. In the second case, there will be no lowering, since $E_{c o l}=E$ ( $E$ - modulus of elasticity of roof rocks). In fact, as a rule, the subsidence of the earth's surface is observed at a rate equal to $0.8-0.9$ from the extracted thickness of a seam $h$ (possibly partially and roof rocks). To obtain such a result in numerical modeling, it is necessary to obtain the corresponding value of the modulus of elasticity of the $E_{c o l}$ for collapsed rocks using the algorithm of sequential division in half of the interval of values $E$. In accordance with the above cases, the initial interval $-0 \div E$. With this division, for further analysis, the halfinterval (either $0 \div E / 2$ or $E / 2 \div E$ ) is taken, on one side of which the sedimentation values will be less than $0.8 h$, and on the other more than $0.8 h$. This half-interval is again divided in half, etc. As a result, we obtain the value of $E_{c o l}$, at which the subsidence of the earth's surface is $0.8 \mathrm{~h}$. In further calculations, this particular feature should be used to model collapsed rocks.

Bilinear plastic model was used in the calculations. In the framework of this model, the material is deformed elastically with the elastic modulus inherent in this undisturbed material, and when the elastic limit (plasticity) is reached, its elastic modulus decreases by 2-3 orders of magnitude. I. e. in fact, the regime of ideal plasticity is realized. Such properties were endowed with rocks of the immediate roof and coal. For coal the limit of plasticity was taken to be equal to $\sigma_{p}=2 \mathrm{MPa}$, and for the rocks of the roof - $\sigma_{p}=4 \mathrm{MPa}$. It should be borne in mind that the values of the parameters characterize the behavior of coal and rocks in the array. In comparison with the values obtained in laboratory experiments, they are many times (orders of magnitude) less, which is determined by the coefficient of structural weakening of the rocks of the array. In the end, the values of the limits of 
plasticity are "effective" values and should be determined by the inverse recalculation when comparing the calculated values and the actually measured parameters in the rock mass. Such parameters can be actually only displacement of rocks of a contour of development (a roof and lateral walls of development). It should be noted here that coal has a clear property of flow, which leads over time to a significant change in the shape of the cross section of the workings. This is very important in the case of the early creation of a DC, which can be in a state of waiting up to several months.

Creep parameters are also unknown and cannot be pre-determined in the laboratory. Nevertheless, it is known that the soil heaving in the workings passed on coal can be considered to be $\sim 30 \mathrm{~cm} /$ month. Based on this value, the corresponding creep parameters are found in the solution of the inverse problem. In the calculations it was assumed that the creep rate $\varepsilon=$ const. This is the simplest option, which allows to display to some extent the time changes of deformation processes in the coal massif. This model is not applicable to describe the process of rooted creep process leading to catastrophic destruction of workings, but it is in the considered period of time and does not occur in reality.

\section{Simulation}

The problem is solved by the finite element method. The entire calculated area is a rectangle with dimensions of $70 \times 60 \mathrm{~m}$. the Boundary conditions are defined as follows: at the lateral vertical boundaries of the calculated area, the values of horizontal stresses corresponding to the lateral spacer are set, while the vertical degree of freedom is not limited; at the lower boundary - there are zero horizontal and vertical displacements; the upper boundary, which is the day surface, is completely free. In addition, as a load applied to the computational domain - the force of gravity.

The General principle of construction of numerical finite element models requires that the calculated area in size was $3 \div 4$ larger than the characteristic size of the simulated objects, so that there was no force influence on the boundaries of the region. In this case, one of the boundaries, namely the earth's surface, is present in the zone of influence of the developed space and cannot be attributed to the required distance. In this regard, it must be included in the calculation scheme explicitly, as one of the boundaries of the computational domain with the corresponding boundary conditions.

It is known that in this formulation of the problem, the resulting voltage solutions correspond to real values, including both the initial voltage in the intact array, and additional resulting from the formation of workings. As for the displacements in the array, both in the reservoir and in the lateral rocks, they are calculated incorrectly. They can be corrected by subtracting from them some fictitious values of the displacements obtained in the solution of the problem in the formulation of the so-called "Greenfield". This solution is for the same computational domain and boundary conditions, but without workings. Similarly, the adjusted offsets will already correspond to the real ones.

As for the deformation properties of rocks and coal, the following values were taken: coal - modulus of elasticity $E=3 * 10^{3} \mathrm{MPa}$, Poisson's ratio $v=0.35$, density $\rho=1300 \mathrm{~kg} / \mathrm{m}^{3}$; lateral rocks $-E=2.3 * 10^{4} \mathrm{MPa}, v=0.27, \rho=2700 \mathrm{~kg} / \mathrm{m}^{3}$. In this case, it is possible to include in the calculation of the layers of different rocks in the roof of the formation (with different deformation properties), modeling the immediate, the main roof and other possible layers and interlayers.

Consider the development of mechanical situation, when DC is created in the process of moving the complex to the end point of excavation site. At the same time, there is a continuous increase in the developed space and the last open pit in which the combine is located, and is part of the future DC, which is further expanded to the full size of the DC. 
When modeling, the problem was solved in full stresses, i.e., the loading of the even region was carried out by gravity under given constraints on the external boundaries of this region. In this formulation, quite correct stresses are obtained at all points of the region, but the displacements must be adjusted by the value of the "green field" displacements. Fig. 1 shows several characteristic cross sections by which the distributions of the parameters of the stress-strain state of the array at the time of DC creation were calculated.

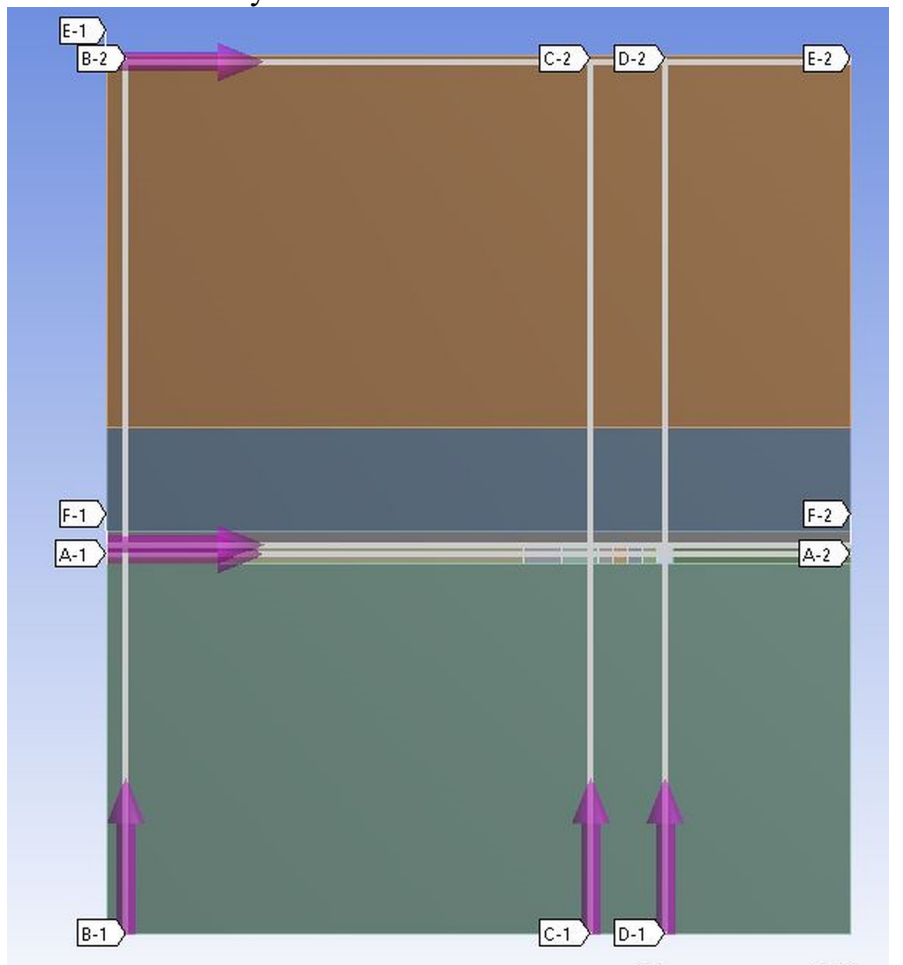

Fig. 1. Characteristic cross sections for calculation of stress-strain state parameters.

Section A1-A2 passes in the middle of the coal seam, section F1-F2 - in close proximity to the formation in its roof. Vertical sections B1-B2 and C1-C2 can be used to calculate "Green field" offsets.

Fig. 2 shows the values of these offsets. At the level of the formation, they undergo a jump due to the softness of coal in comparison with the rocks of the roof and soil. For the selected configuration of the computational domain on the day surface, they are $\sim 2.89 \mathrm{~cm}$, and at the level of the formation roof $\sim 2.1 \mathrm{~cm}$. Note that the obtained values due to the setting of the back-Chi are fictitious values that do not exist in real conditions. On the layer the displacement jump is equal to $4 \mathrm{~mm}$.

Note that the distributions of displacements in B1-B2 and $\mathrm{C} 1-\mathrm{C} 2$ are almost the same (shown in green and red), which should be expected in the absence of mass workings.

The nature of the distribution of the vertical calculated displacements in the entire region is shown in fig. 2. To obtain real values, they must be adjusted according to the curves in fig. 3. The presented picture is mainly illustrative, since the main interest is the displacement in the immediate vicinity of the DC, at its borders. At this level, the adjustment value according is $\sim 2.0 \mathrm{~cm}$. Note that in accordance with what was said earlier, the modulus of elasticity over developed-ness of the space was taken to be equal $8 * 10^{2}$ $\mathrm{MPa}$. in comparison with the module for the overlying rock mass $\mathrm{E}=2.3^{*} 10^{4} \mathrm{MPa}$, i.e. almost 30 less. 
Fig. 4 shows the distribution of vertical displacements in the horizontal sections A1-A2 (red) and F1-F2 (green). The results show that the rocks collapsed in the developed space shrank under the influence of mountain pressure by $\sim 14 \mathrm{~cm}$, since the rocks in the soil of the formation are practically not deformed.

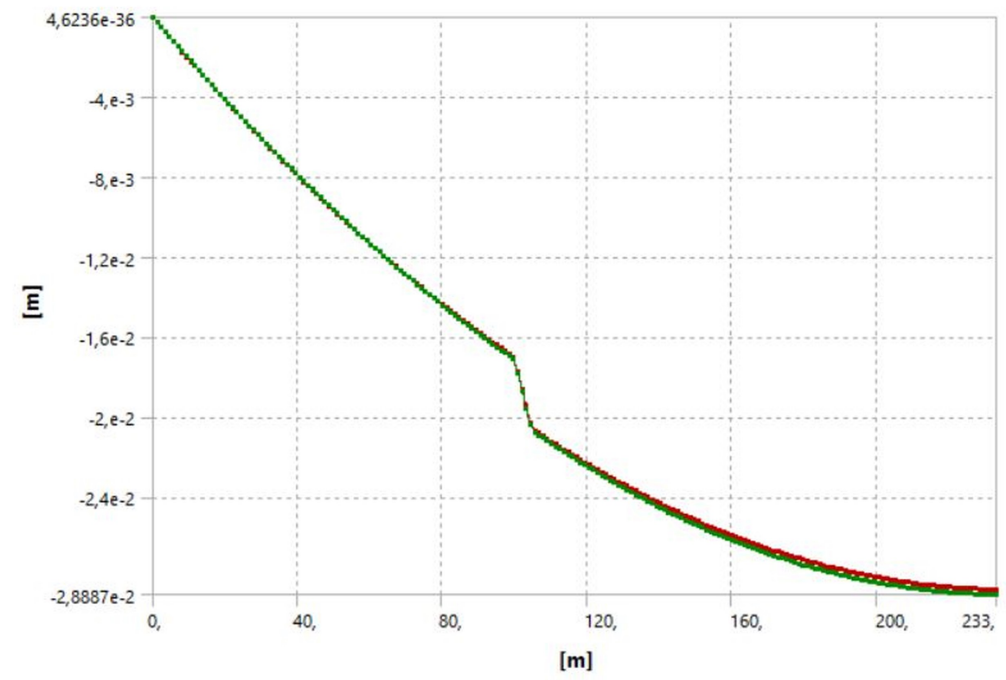

Fig. 2. Vertical displacements in sections B1-B2 and C1-C2.

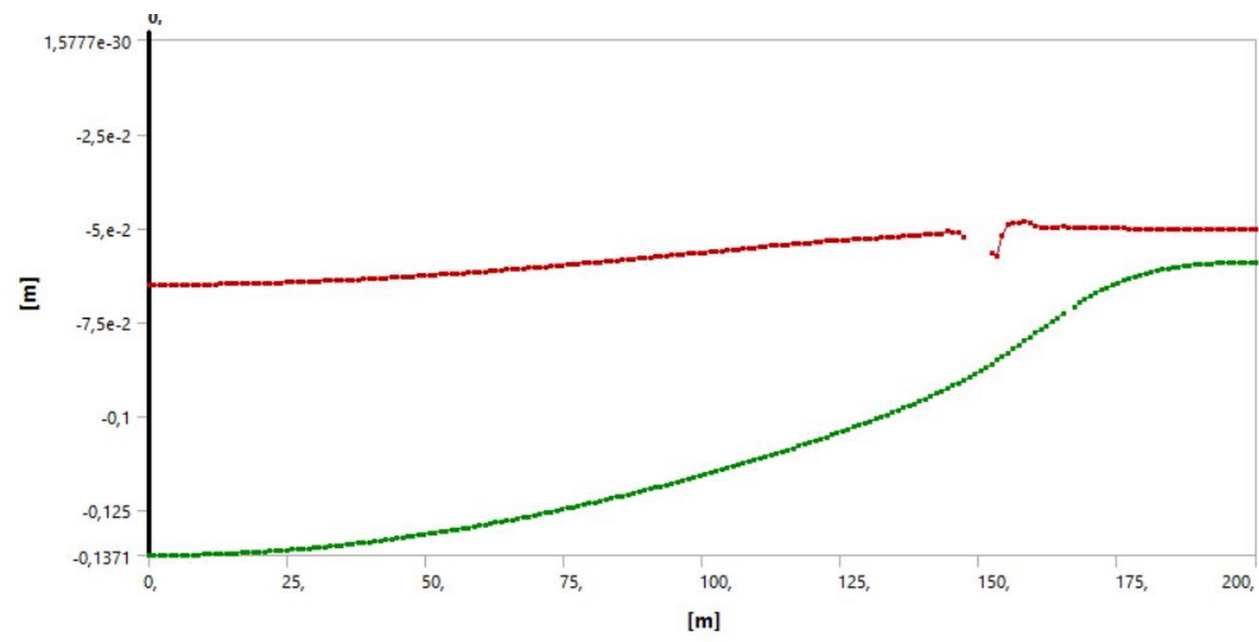

Fig. 4. Vertical displacements along horizontal cross sections.

Fig. 5 shows the distribution of horizontal displacements near DC. As expected, they are directed inside the mine and from the coal seam on the vertical wall of the mine are $\sim 17 \mathrm{~cm}$.

Consider now (Fig. 6) stress distribution over previously selected sections A1-A2 (red) and F1-F2 (green). Note that for the selected parameters of development, i.e. the depth of the reservoir in $130 \mathrm{~m}$ and the average density of the overlying rocks in $2.7 \mathrm{t} / \mathrm{m}^{3}$, the vertical stresses are $\sim 3.51 \mathrm{MPa}$. The horizontal asymptotes of the above distributions coincide with this value. The peak at $x=150 \mathrm{~m}$ for the section above the formation roof corresponds to the voltage drop directly above the production. At the same time, at some distance from the output $(\sim 10 \mathrm{~m})$, the reference pressure maxima with a concentration of 1.2 are observed. 
The distribution of vertical stresses around DC is shown in fig. 7. In the roof and the soil of the development are visible discharge zone (the level of unloading is shown in shades of red). Near the roof there are minor zones of tensile stresses. In the horizontal direction there are also unloading zones up to tensile stresses.

A series of calculations refers to the option of creating DC tunneling combine at the end of working off the column. Similar calculations were previously carried out for the case of a previously passed DC [7].

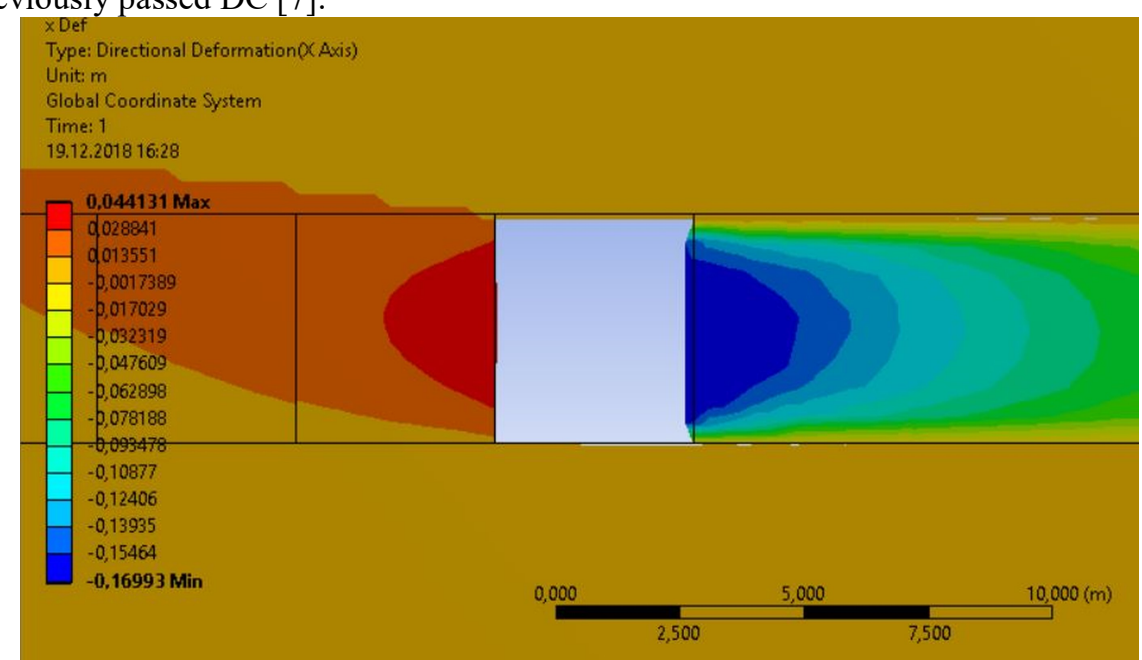

Fig. 5. Horizontal displacements near DC.

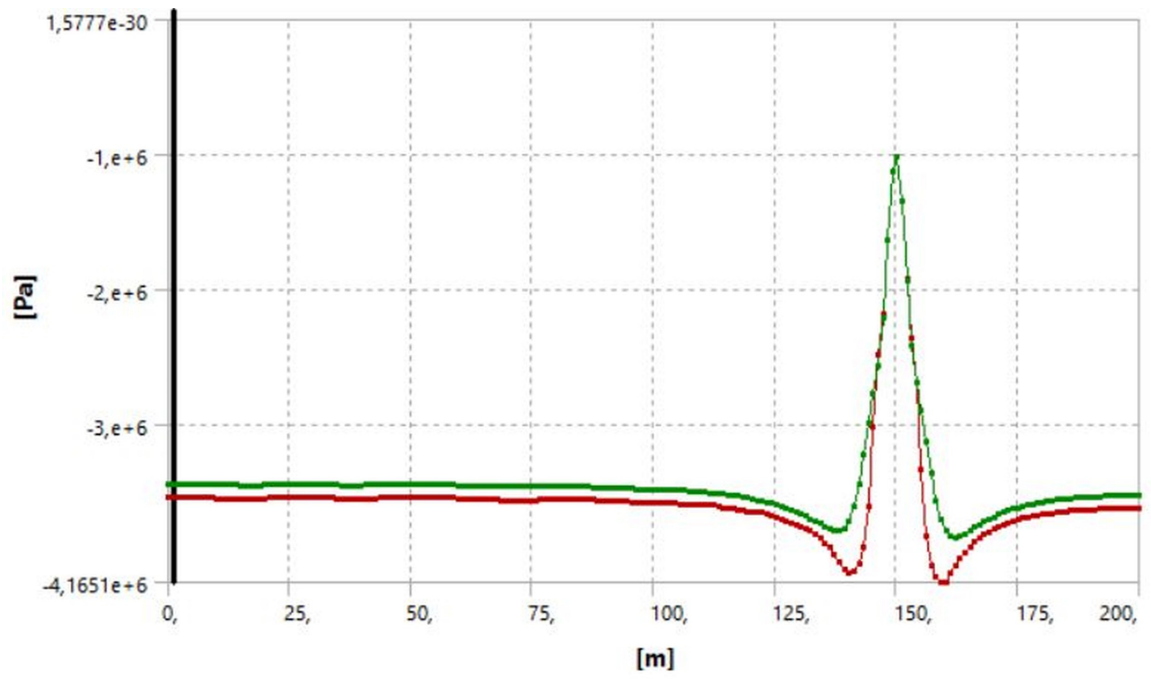

Fig. 6. Distribution of vertical stresses in the middle of the formation.

\section{Conclusion.}

In conclusion, we note that taking into account the plastic deformation of rocks and coal allows by calculation to obtain comparable to the real values of the subsidence of the roof into pre-formed dismantling the camera. Conducting observations of the actual lowering of 
the roof, it is possible to establish with certain accuracy the value of the plasticity limit for the coal of this formation, and use it in the future.

Comparing both variants of DC formation, it can be noted that in the variant of TRANS-temporal sinking of DC, the pillar between the treatment output and DC at the last stage is almost completely destroyed forming a single space from the bottom-hole work, the DC and the non - load-bearing pillar. From the position of mechanics such a development of events, namely, almost a one-time increase in the span of the hanging roof in $2 \div 3$ times can lead to a significant increase in stresses in the array and an increase in the concentration of vertical stresses on the DC wall opposite to the approaching face. In addition, the long standing of the chamber is associated with manifestations of creep of coal and rocks, which ultimately manifests itself in the collapse of the roof and walls, or in reducing the crosssection of the chamber to unacceptable values. Most of this kind of adverse manifestations of mountain pressure is deprived of the system with the formation of DC tunneling machine in the process of refining the post.
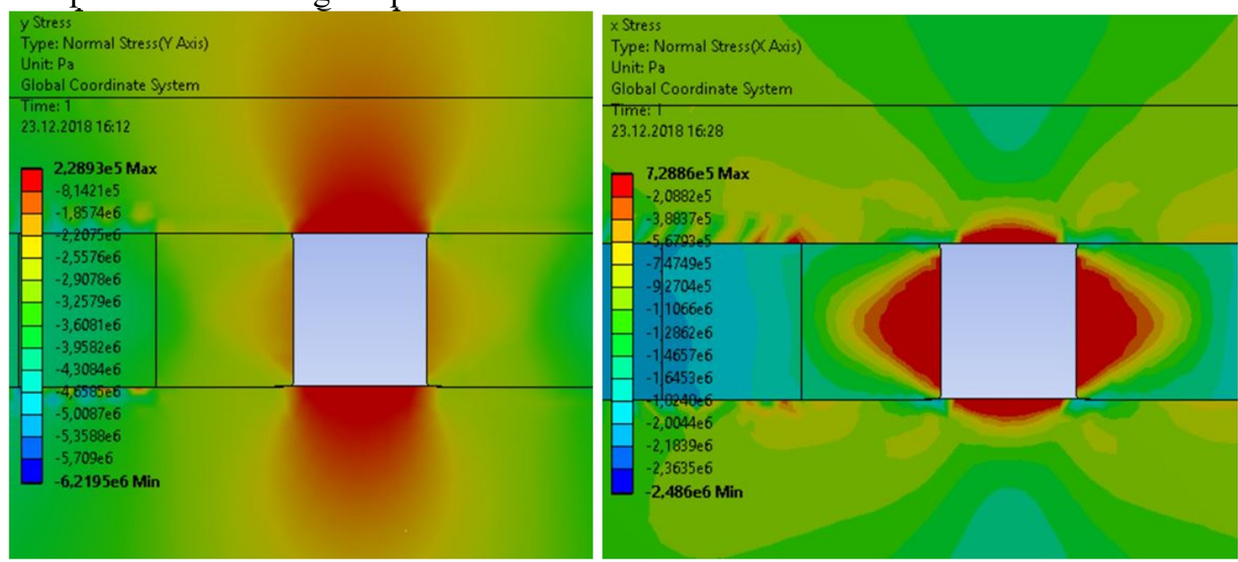

Fig. 7. Distribution of vertical (left) and horizontal (write) stresses around DC.

\section{References}

1. A. M. Nikolsky, A. A. Neverov, S. A. Neverov, M. V. Shinkevich, J. Min. Sci., 69, 5 (2008)

2. A. A. Gedanken, N. Zhdankin, J. Min. Sci., 4, 3 (1985)

3. G. A. Katkov, J. Iss. Min. Pres., 2, 37 (1979)

4. F. V. Remezov, S. V. Novoselov, Ugol, 6, 23 (2018)

5. V. O. Torro, A. V. Remezov, V. V. Klimov, Y. A. Dedikov, KuzsTU Bulletin, 6, 67 (2017)

6. K. N. Trubetskoi, M. A. Iofis, S. V. Kuznetsov, V. A. Trofimov, J. Min. Sci., 35, 3 (1999)

7. V. A. Trofimov, S. S. Kubrin, Y. A. Filippov, I. L. Kharitonov, Mining informational and analytical bulletin, 11, 48, (2018) 